\title{
Approximations of the Sixth Order with the Polynomial and Non-polynomial Splines and Variational-difference Method
}

\author{
I. G. Burova
}

\begin{abstract}
This paper discusses the approximations with the local basis of the second level and the sixth order. We call it the approximation of the second level because in addition to the function values in the grid nodes it uses the values of the function, and the first and the second derivatives of the function. Here the polynomial approximations and the non-polynomial approximations of a special form are discussed. The non-polynomial approximation has the properties of polynomial and trigonometric functions. The approximations are twice continuously differentiable. Approximation theorems are given. These approximations use the values of the function at the nodes, the values of the first and the second derivatives of the function at the nodes, and the local basis splines. These basis splines are used for constructing variational-difference schemes for solving boundary value problems for differential equations. Numerical examples are given
\end{abstract}

Keywords-Non-polynomial approximation, polynomial approximation, variational-difference method.

\section{INTRODUCTION}

$\mathrm{T}$ HE advantages of finite-difference methods for the approximate solution of boundary value problems for differential equations are well known. The finite difference matrix contains few nonzero elements. The elements of the finite difference matrix, as well as the right-hand side of this system, are calculated quite simply. Variational difference methods have several advantages over the finite-difference methods. For example, there are cases when the difference approximation of an elliptic differential equation is nonelliptic. A lot of attention to the construction and study of variational-difference methods was given by Prof. S.G.Michlin (see [1]). In the paper, the construction of polynomial splines of the Hermitian type was considered. Prof. S.G.Mikhlin constructed variational-difference approximations based on these splines. In recent years variational-difference schemes are within the area of focus of some papers (see, for example, [2], [3].

$\mathrm{B}$-splines can be used to solve boundary value problems. In study [4] the authors deal with the cubic B-spline method to solve two-point boundary value problems. The cubic B-spline approximation equation, based on quarter-sweep concept, are

I. G. Burova is with the St. Petersburg State University, 7/9 Universitetskaya nab., St.Petersburg, 199034 Russia (corresponding author, email: burovaig@mail.ru,i.g.burova@mail.ru). used to discretize the proposed problem and construct the linear system. For example, the variational-difference method and B-spline interpolation method with different degrees were used in paper [5] for the design of the lenses surfaces. Hermite-type splines make it possible to approximate not only a function, but also its derivatives. Hermite splines are often used to solve various problems (see [6]). Much attention is paid to the construction of splines in the papers by prof. Y.K.Demjanovich (see [7]). Among non-polynomial splines, trigonometric splines are of significant interest. Cubic Hermitian splines are widely known and often used. Cubic Hermite curves are adopted in conjunction with the level set method to represent curved interfaces in paper [8].

In paper [9] the authors construct spline interpolation with the property of monotonicity and convexity preservation, using two types of splines: the Cubic Spline (CS), and the Hermite Cubic Rational polynomial Spline (CRS). Both curves are based on the shape preserving the Hermite Variable Degree Spline (VDS).

In 1964 Schoenberg introduced trigonometric spline functions and proved the existence of locally supported trigonometric spline and B-spline functions [10]. In some cases, the use of trigonometric splines is preferable to the use of polynomial splines.

This paper continues the series of papers on approximation with local polynomial and non-polynomial splines (see [11], [12], [13], [14], [15]). The proposed paper offers nonpolynomial splines of the Hermite type with the sixth order approximation of the second level (height), as well as smooth non-polynomial splines. The construction of these splines uses the functions of the Chebyshev system. These nonpolynomial splines solve the Hermite interpolation problem. These local basis functions can be used in solving problems of the mean-square approximation, solving boundary value problems by the variational-difference method, and solving integral equations. In this paper we consider the features of the application of polynomial and non-polynomial splines of the sixth order of approximation of the second level for solving boundary value problems by the variational-difference method. The advantages of using sixth-order splines of approximation of the second height include the fact that we obtain not only an approximate solution, but also approximations to the first and second derivatives of the solution. 


\section{AbOut VARIATIONAL-DifFERENCE METHOD}

\section{A. General Information}

First, we recall the features of solving boundary value problems by the variational-difference method.

Denote by $A$ a positive definite symmetric operator in a real Hilbert space $H$. Thus, there exists a constant $\gamma$ such that the inequality

$$
(A u, u) \geq \gamma(u, u), \gamma>0, u \in D(A)
$$

is valid where $D(A)$ is the domain of definition of the operator $A$ dense in $H$. Consider the equation

$$
A u=f, f \in H \text {. }
$$

The bilinear form

$$
[u, v]=(A u, v), u, v \in D(A)
$$

defines a scalar product on $D(A)$. Denote by $H_{A}$ the completion of $D(A)$ with respect to the norm generated by the scalar product (3). Applying inequality (1) we see that for $u_{n}, u_{m} \in D(A)$ the followed inequality valid

$$
\left[u_{n}-u_{m}, u_{n}-u_{m}\right] \geq \gamma\left(u_{n}-u_{m}, u_{n}-u_{m}\right) .
$$

Thus, the convergence in $H_{A}$. implies the convergence in $H$. We have a chain of embeddings $D(A) \subset H_{A} \subset H$. From the inequality (1) it is easy to obtain the following inequality

$$
[u, u] \geq \gamma(u, u), u \subset H_{A} .
$$

We denote the energy functional by $F(u)$,

$$
F(u)=[u, u]-2(u, f), u \in H_{A} .
$$

It is known that if equation (2) has a solution, then the functional $F(u)$ has the smallest value when $u=u_{0}$. The converse is also true, the element $u_{0}$ at which the minimum of functional $F(u)$ is reached is a solution to equation (2). Thus, if $u_{0}$. is an element on which the minimum of the energy functional is realized, then we obtain the equation:

$$
\left[u_{0}, v\right]=(f, v), \quad v \in H_{A} .
$$

Let space $H^{(n)}$ be such that $H^{(n)} \subset H_{A}$. Let a basis $\varphi_{1}, \ldots, \varphi_{n}$ be chosen in the space $H^{(n)}$. We take an approximate solution $U, U \in H^{(n)}$, in the form:

$$
U=\sum_{i=1}^{n} c_{i} \varphi_{i}, \quad c_{i}=\text { const }
$$

Now we receive

$$
F(U)=\left[\sum_{i=1}^{n} c_{i} \varphi_{i}, \sum_{j=1}^{n} c_{j} \varphi_{j}\right]-2\left(f, \sum_{j=1}^{n} c_{j} \varphi_{j}\right)
$$

From the condition

$$
\frac{\partial F(U)}{\partial c_{i}}=0, \quad i=1,2, \ldots, n,
$$

We obtain the system of equations:

$$
\sum_{i=1}^{n} c_{i}\left[\varphi_{i}, \varphi_{j}\right]=\left(f, \varphi_{j}\right), j=1,2, \ldots, n
$$

Using the method described above, we will solve the boundary problem:

$$
\begin{gathered}
A u=-\frac{d}{d x}\left(p(x) \frac{d u}{d x}\right)+r(x) u=f(x), \\
u(a)=0, u(b)=0 .
\end{gathered}
$$

In the case of setting the boundary conditions $u(a)=0, u(b)=0$, the scalar product can be determined by the formula (see [1]):

$$
\begin{gathered}
(A u, v)=\int_{a}^{b}\left(p(x) \frac{d u}{d x} \frac{d v}{d x}+r(x) u(x) v(x)\right) d x . \\
(u, f)=\int_{a}^{b} u f d x .
\end{gathered}
$$

To solve the boundary value problem, we apply the variational-difference method while using sixth-order polynomial splines of approximation of the second level. In the next section, polynomial and non-polynomial approximations will be considered; a numerical example will be considered in the fourth section.

\section{ABOUT SIXTH ORDER SPLINE APPROXIMATION}

Let $n$ be a positive number, $n>3$, and $a, b$ real numbers. Let function $u(x)$ be such that $u \in C^{6}([a, b])$. The nodes $x_{j} \in[a, b], \quad j=0, \ldots, n$, such that $a \leq \cdots<x_{j-1}<x_{j}<$ $x_{j+1}<\ldots \leq b$. The formulas of the basis splines of the second level and the sixth order of approximation $w_{j, 0}(x)$, $w_{j+1,0}(x), \quad w_{j, 1}(x), \quad w_{j+1,1}(x), \quad w_{j, 2}(x), \quad w_{j+1,2}(x), \quad$ on an interval $\left[x_{j}, x_{j+1}\right]$

are obtained by solving the following system of equations:

$$
\begin{gathered}
\varphi_{i}\left(x_{i}\right) w_{j, 0}(x)+\varphi_{i}\left(x_{i+1}\right) w_{j+1,0}(x)+ \\
\varphi^{\prime}{ }_{i}\left(x_{i}\right) w_{j, 1}(x)+\varphi^{\prime}{ }_{i}\left(x_{i+1}\right) w_{j+1,1}(x)+ \\
\varphi^{\prime \prime}{ }_{i}\left(x_{i}\right) w_{j, 2}(x)+\varphi^{\prime \prime}{ }_{i}\left(x_{i+1}\right) w_{j+1,2}(x)=\varphi_{i}(x), \\
i=0,1,2,3,4,5 .
\end{gathered}
$$

The system of functions $\varphi_{i}$ should be the Chebyshev system on the interval $[\alpha, \beta]$, where $\alpha, \beta$ are real numbers, $\alpha<\beta$.

Based on different systems $\varphi_{i}$ we will obtain different basis functions $w_{j, 0}(x), w_{j+1,0}(x), w_{j, 1}(x), w_{j+1,1}(x), w_{j, 2}(x)$, $w_{j+1,2}(x)$. We construct the approximation of function $U(x)$ with these splines on the interval $\left[x_{j}, x_{j+1}\right]$ in the form: 
$U(x)=u\left(x_{j}\right) w_{j, 0}(x)+u\left(x_{j+1}\right) w_{j+1,0}(x)+u^{\prime}\left(x_{j}\right) w_{j, 1}(x)+$ $+u^{\prime}\left(x_{j+1}\right) w_{j+1,1}(x)+u^{\prime \prime}\left(x_{j}\right) w_{j, 2}(x)+u^{\prime \prime}\left(x_{j+1}\right) w_{j+1,2}(x)$.

The following theorem is valid if $\varphi_{i}=x^{i}, \quad i=$ $0,1,2,3,4,5$.

Theorem 1. Let function $u(x)$ be such that $u \in \mathrm{C}^{6}([a, b])$. $\varphi_{i}=x^{i}, i=0,1,2,3,4,5$. Suppose the ordered distinct nodes $x_{k}$ are $x_{k+1}-x_{k}=h$. Then for $x \in\left[x_{j}, x_{j+1}\right]$ we have

$$
|u(x)-U(x)| \leq K_{1} h^{6}\left\|u^{(6)}\right\|_{\left[x_{j}, x_{j+1}\right]}
$$

$$
K_{1} \approx 0.015625 / 6 \text { ! . }
$$

Proof. On the interval $\left[x_{j}, x_{j+1}\right]$ we have the following relations: $U\left(x_{j}\right)=u\left(x_{j}\right), U^{\prime}\left(x_{j}\right)=u^{\prime}\left(x_{j}\right), U^{\prime \prime}\left(x_{j}\right)=u^{\prime \prime}\left(x_{j}\right)$, $U\left(x_{j+1}\right)=u\left(x_{j+1}\right), U^{\prime \prime}\left(x_{j+1}\right)=u^{\prime \prime}\left(x_{j+1}\right), \quad U^{\prime}\left(x_{j+1}\right)=$ $u^{\prime}\left(x_{j+1}\right)$. Thus we can construct the Hermite interpolation polynomial $U(x)$ on the interval $\left[x_{j}, x_{j+1}\right]$ with the basis functions:

$w_{j, 0}(x)=\frac{\left(x-X_{j+1}\right)^{3}}{\left(X_{j}-X_{j+1}\right)^{5}} P_{2 j}$, where $x \in\left[X_{j}, X_{j+1}\right]$,

$P_{2 j}=6 x^{2}+3 x X_{j+1}-15 x X_{j}+10 X_{j}^{2}+X_{j+1}^{2}-5 X_{j} X_{j+1}$,

$w_{j, 0}(x)=\frac{\left(x-X_{j-1}\right)^{3}}{\left(X_{j}-X_{j-1}\right)^{5}} Q_{2 j}$, where $x \in\left[X_{j-1}, X_{j}\right]$,

$Q_{2 j}=6 x^{2}+3 x X_{j-1}-15 x X_{j}+10 X_{j}^{2}+X_{j-1}^{2}-5 X_{j} X_{j-1}$,

$w_{j+1,0}(x)=\frac{-\left(x-X_{j}\right)^{3}}{\left(X_{j}-X_{j+1}\right)^{5}} S_{2 j}$, where $x \in\left[X_{j}, X_{j+1}\right]$,

$S_{2 j}=6 x^{2}+3 x X_{j}-15 x X_{j+1}+X_{j}^{2}+10 X_{j+1}^{2}-5 X_{j} X_{j+1}$,

$w_{j, 1}(x)=\frac{-\left(x-X_{j+1}\right)^{3}}{\left(X_{j}-X_{j+1}\right)^{4}}\left(x-X_{j}\right) P_{1 j}$, where $x \in\left[X_{j}, X_{j+1}\right]$,

$P_{1 j}=3 x-4 X_{j}+X_{j+1}$,

$w_{j, 1}(x)=\frac{-\left(x-X_{j-1}\right)^{3}}{\left(X_{j}-X_{j-1}\right)^{4}}\left(x-X_{j}\right) Q_{1 j}$, where $x \in\left[X_{j-1}, X_{j}\right]$,

$Q_{1 j}=3 x-4 X_{j}+X_{j-1}$

$w_{j+1,1}(x)=\frac{-\left(x-X_{j}\right)^{3}}{\left(X_{j}-X_{j+1}\right)^{4}}\left(x-X_{j+1}\right) S_{1 j}$, where $x \in\left[X_{j}, X_{j+1}\right]$,

$S_{1 j}=3 x-4 X_{j+1}+X_{j}$,

$w_{j, 2}(x)=\frac{\left(x-X_{j+1}\right)^{3}}{2\left(X_{j}-X_{j+1}\right)^{3}}\left(x-X_{j}\right)^{2}$, where $x \in\left[X_{j}, X_{j+1}\right]$,

$w_{j, 2}(x)=\frac{\left(x-X_{j-1}\right)^{3}}{2\left(X_{j}-X_{j-1}\right)^{3}}\left(x-X_{j}\right)^{2}$, where $x \in\left[X_{j-1}, X_{j}\right]$,

$w_{j+1,2}(x)=\frac{-\left(x-X_{j+1}\right)^{2}}{2\left(X_{j}-X_{j+1}\right)^{3}}\left(x-X_{j}\right)^{3}$, where $x \in\left[X_{j}, X_{j+1}\right]$,

These basis functions can be obtained from the system of equations, when $\varphi_{i}=x^{i}$.

Using the theorem of the error of Hermite interpolation we get that the error of the interpolation will be the following:
$u(x)-U(x)=\frac{u^{(6)}(\xi)}{6 !}\left(x-x_{j}\right)^{3}\left(x-x_{j+1}\right)^{3}, \xi=\xi(x)$,

$\xi \in\left[x_{j}, x_{j+1}\right]$.

If we put $x=x_{j}+t h, t \in[0,1]$, we obtain

$\left(x-x_{j}\right)^{3}\left(x-x_{j+1}\right)^{3}=h^{6} t^{3}(t-1)^{3}$. It is easy to obtain $\max _{t \in[0,1]}\left|t^{3}(t-1)^{3}\right|=0.015625$.

The proof is complete.

Remark. If $x=x_{j}+t h, t \in[0,1]$, then the basis splines are of the form:

$$
\begin{gathered}
w_{j, 0}\left(x_{j}+t h\right)=-\left(6 t^{2}+3 t+1\right)(t-1)^{3}, \\
w_{j+1,0}\left(x_{j}+t h\right)=\left(6 t^{2}-15 t+10\right) t^{3}, \\
w_{j, 1}\left(x_{j}+t h\right)=-t h(3 t+1)(t-1)^{3}, \\
w_{j+1,1}\left(x_{j}+t h\right)=-h(t-1)(3 t-4) t^{3}, \\
w_{j, 2}\left(x_{j}+t h\right)=-h^{2} t^{2}(t-1)^{3} / 2, \\
w_{j+1,2}\left(x_{j}+t h\right)=h^{2} t^{3}(t-1)^{2} / 2,
\end{gathered}
$$

Solving the system of equations when $\varphi_{s}=\cos (s x), s=$ $0,1,2, \quad \varphi_{s}=\sin (s x), \quad s=1,2, \quad \varphi_{5}=x$, we obtain the formulas of nonpolynomial basis splines of the sixth order of approximation of the second level:

$$
\begin{aligned}
& W_{j, 0}=\left(16 X_{j+1}-16 x+15 \sin \left(X_{j}-X_{j+1}\right)\right. \\
& -12 \sin \left(2 X_{j}-2 X_{j+1}\right)+8 \sin \left(x-X_{j}\right) \\
& +18\left(x-X_{j+1}\right) \cos \left(X_{j}-X_{j+1}\right)+ \\
& 2\left(X_{j+1}-x\right) \cos \left(3 X_{j}-3 X_{j+1}\right)+3 \sin \left(3 X_{j}-3 X_{j+1}\right) \\
& -12 \sin \left(x+X_{j}-2 X_{j+1}\right)+4 \sin \left(x-3 X_{j}+2 X_{j+1}\right) \\
& -12 \sin \left(x-2 X_{j}+X_{j+1}\right)+4 \sin \left(x-3 X_{j+1}+2 X_{j}\right) \\
& +4 \sin \left(2 x-2 X_{j}\right)-6 \sin \left(2 x-X_{j}-X_{j+1}\right) \\
& -\sin \left(2 x-3 X_{j}+X_{j+1}\right)+4 \sin \left(2 x-2 X_{j+1}\right) \\
& \left.-\sin \left(2 x+X_{j}-3 X_{j+1}\right)+8 \sin \left(x-X_{j+1}\right)\right) / d \text {, } \\
& d=2\left(8\left(X_{j+1}-X_{j}\right)+15 \sin \left(X_{j}-X_{j+1}\right)\right. \\
& -12 \sin \left(2 X_{j}-2 X_{j+1}\right)+9\left(X_{j}-X_{j+1}\right) \cos \left(X_{j}-X_{j+1}\right) \\
& +\left(X_{j+1}-X_{j}\right) \cos \left(3 X_{j}-3 X_{j+1}\right)+3 \sin \left(3 X_{j}-3 X_{j+1}\right) \text {, } \\
& W_{j+1,0}=\left(18\left(X_{j}-x\right) \cos \left(X_{j}-X_{j+1}\right)\right. \\
& +2\left(x-X_{j}\right) \cos \left(3 X_{j}-3 X_{j+1}\right)+3 \sin \left(3 X_{j}-3 X_{j+1}\right) \\
& +12 \sin \left(x+X_{j}-2 X_{j+1}\right)+12 \sin \left(x+X_{j+1}-2 X_{j}\right) \\
& -4 \sin \left(x-3 X_{j}+2 X_{j+1}\right)-4 \sin \left(x-3 X_{j+1}+2 X_{j}\right) \\
& -4 \sin \left(2 x-2 X_{j}\right)-4 \sin \left(2 x-2 X_{j+1}\right) \\
& +\sin \left(2 x-3 X_{j}+X_{j+1}\right)+\sin \left(2 x-3 X_{j+1}+X_{j}\right) \\
& +6 \sin \left(2 x-X_{j}-X_{j+1}\right)-12 \sin \left(2 X_{j}-2 X_{j+1}\right) \\
& -8 \sin \left(x-X_{j+1}\right)-8 \sin \left(x-X_{j}\right)+16\left(x-X_{j}\right) \\
& +15 \sin \left(X_{j}-X_{j+1}\right) / d \text {, } \\
& W_{j, 1}=\left(9 \cos \left(3 X_{j}-3 X_{j+1}\right)-9 \cos \left(X_{j}-X_{j+1}\right)\right. \\
& +3 \cos \left(X_{j}-3 X_{j+1}+2 x\right) \\
& +24\left(X_{j}-X_{j+1}\right) \sin \left(x+X_{j+1}-2 X_{j}\right) \\
& +2\left(X_{j}-X_{j+1}\right) \sin \left(X_{j}-3 X_{j+1}+2 x\right) \\
& +8\left(X_{j+1}-X_{j}\right) \sin \left(x-3 X_{j+1}+2 X_{j}\right) \\
& +6\left(X_{j}-X_{j+1}\right) \sin \left(2 x-X_{j}-X_{j+1}\right)
\end{aligned}
$$




$$
\begin{aligned}
& +12 \cos \left(x+X_{j+1}-2 X_{j}\right)+12 \cos \left(x+X_{j}-2 X_{j+1}\right) \\
& -12 \cos \left(x-3 X_{j+1}+2 X_{j}\right)-12 \cos \left(x-3 X_{j}+2 X_{j+1}\right) \\
& +24\left(X_{j}-x\right) \sin \left(2 X_{j}-2 X_{j+1}\right) \\
& +8\left(X_{j+1}-X_{j}\right) \sin \left(2 x-2 X_{j}\right) \\
& +6\left(x-X_{j+1}\right) \sin \left(3 X_{j}-3 X_{j+1}\right) \\
& +16\left(X_{j+1}-X_{j}\right) \sin \left(x-X_{j}\right) \\
& +\sin \left(X_{j}-X_{j+1}\right)\left(30 x-18 X_{j+1}-12 X_{j}\right) \\
& \left.-6 \cos \left(2 x-X_{j}-X_{j+1}\right)+3 \cos \left(2 x-3 X_{j}+X_{j+1}\right)\right) /(2 d) \text {, } \\
& W_{j+1,1}=\left(9 \cos \left(X_{j}-X_{j+1}\right)-9 \cos \left(3 X_{j}-3 X_{j+1}\right)\right. \\
& -3 \cos \left(X_{j}-3 X_{j+1}+2 x\right)+16\left(X_{j+1}-X_{j}\right) \sin \left(x-X_{j+1}\right) \\
& +6\left(X_{j}-X_{j+1}\right) \sin \left(2 x-X_{j}-X_{j+1}\right) \\
& +\sin \left(X_{j}-X_{j+1}\right)\left(30 x-12 X_{j+1}-18 X_{j}\right) \\
& +8\left(X_{j+1}-X_{j}\right) \sin \left(2 x-2 X_{j+1}\right) \\
& -12 \cos \left(x+X_{j+1}-2 X_{j}\right)+12 \cos \left(x-3 X_{j+1}+2 X_{j}\right) \\
& +6 \cos \left(2 x-X_{j}-X_{j+1}\right)-3 \cos \left(2 x+X_{j+1}-3 X_{j}\right) \\
& +6\left(x-X_{j}\right) \sin \left(3 X_{j}-3 X_{j+1}\right) \\
& +24\left(X_{j}-X_{j+1}\right) \sin \left(x+X_{j}-2 X_{j+1}\right) \\
& +8\left(X_{j+1}-X_{j}\right) \sin \left(x-3 X_{j}+2 X_{j+1}\right) \\
& +2\left(X_{j}-X_{j+1}\right) \sin \left(2 x-3 X_{j}+X_{j+1}\right) \\
& +24\left(X_{j+1}-x\right) \sin \left(2 X_{j}-2 X_{j+1}\right) \\
& +12 \cos \left(x-3 X_{j}+2 X_{j+1}\right)-12 \cos \left(x+X_{j}-2 X_{j+1}\right) /(2 d) \text {, } \\
& W_{j, 2}=\left(\left(30 x-12 X_{j}-18 X_{j+1}\right) \cos \left(X_{j}-X_{j+1}\right)\right. \\
& +2\left(x-X_{j+1}\right) \cos \left(3 X_{j}-3 X_{j+1}\right)+16 \sin \left(x-X_{j}\right) \\
& +12\left(X_{j}-x\right) \cos \left(2 X_{j}-2 X_{j+1}\right)-8 \sin \left(x-X_{j+1}\right) \\
& -3 \sin \left(3 X_{j}-3 X_{j+1}\right)-12 \sin \left(x+X_{j}-2 X_{j+1}\right) \\
& -4 \sin \left(x-3 X_{j}+2 X_{j+1}\right)+2 \sin \left(2 x-2 X_{j}\right) \\
& +8 \sin \left(x-3 X_{j+1}+2 X_{j}\right)+\sin \left(2 x-3 X_{j}+X_{j+1}\right) \\
& -12 \sin \left(2 x-X_{j}-X_{j+1}\right)+14 \sin \left(2 x-2 X_{j+1}\right) \\
& -5 \sin \left(X_{j}-3 X_{j+1}+2 x\right)+20\left(X_{j+1}-x\right) \\
& +12\left(X_{j+1}-X_{j}\right) \cos \left(x+X_{j+1}-2 X_{j}\right)-6 \sin \left(2 X_{j}-2 X_{j+1}\right) \\
& +16\left(X_{j}-X_{j+1}\right) \cos \left(x-X_{j}\right)+4\left(X_{j}-X_{j+1}\right) \cos \left(2 x-2 X_{j}\right) \\
& +4\left(X_{j+1}-X_{j}\right) \cos \left(x-3 X_{j+1}+2 X_{j}\right)+21 \sin \left(X_{j}-X_{j+1}\right) \\
& +6\left(X_{j+1}-X_{j}\right) \cos \left(2 x-X_{j}-X_{j+1}\right) \\
& +2\left(X_{j}-X_{j+1}\right) \cos \left(2 x+X_{j}-3 X_{j+1}\right) /(2 d) \text {, } \\
& W_{j+1,2}=\left(\left(18 X_{j}+12 X_{j+1}-30 x\right) \cos \left(X_{j}-X_{j+1}\right)\right. \\
& +2\left(X_{j}-x\right) \cos \left(3 X_{j}-3 X_{j+1}\right)-14 \sin \left(2 x-2 X_{j}\right) \\
& -3 \sin \left(3 X_{j}-3 X_{j+1}\right)-8 \sin \left(x-3 X_{j}+2 X_{j+1}\right) \\
& +12 \sin \left(x+X_{j+1}-2 X_{j}\right)+4 \sin \left(x-3 X_{j+1}+2 X_{j}\right) \\
& +12 \sin \left(2 x-X_{j}-X_{j+1}\right)+5 \sin \left(2 x+X_{j+1}-3 X_{j}\right) \\
& -2 \sin \left(2 x-2 X_{j+1}\right)-\sin \left(X_{j}-3 X_{j+1}+2 x\right) \\
& +6\left(X_{j+1}-X_{j}\right) \cos \left(2 x-X_{j}-X_{j+1}\right)-16 \sin \left(x-X_{j+1}\right) \\
& +4\left(X_{j}-X_{j+1}\right) \cos \left(2 x-2 X_{j+1}\right)+8 \sin \left(x-X_{j}\right) \\
& +12\left(X_{j+1}-X_{j}\right) \cos \left(x+X_{j}-2 X_{j+1}\right)+20 x \\
& +12\left(x-X_{j+1}\right) \cos \left(2 X_{j}-2 X_{j+1}\right)-20 X_{j} \\
& +4\left(X_{j+1}-X_{j}\right) \cos \left(x-3 X_{j}+2 X_{j+1}\right) \\
& +16\left(X_{j}-X_{j+1}\right) \cos \left(x-X_{j+1}\right)+21 \sin \left(X_{j}-X_{j+1}\right) \\
& +2\left(X_{j}-X_{j+1}\right) \cos \left(2 x+X_{j+1}-3 X_{j}\right)
\end{aligned}
$$

$$
\left.-6 \sin \left(2 X_{j}-2 X_{j+1}\right)\right) /(2 d)
$$

Figures 1-9 show the plots of the basis functions $W_{j, i}$ and the first and second derivatives of these functions, when $h=1$.

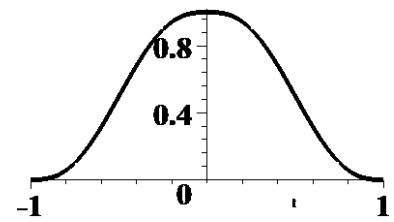

Fig.1. The plot of the $W_{j, 0}$

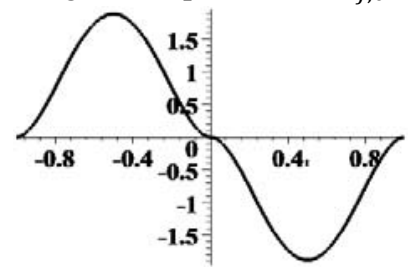

Fig.2. The plot of the $W^{\prime}{ }_{j, 0}$

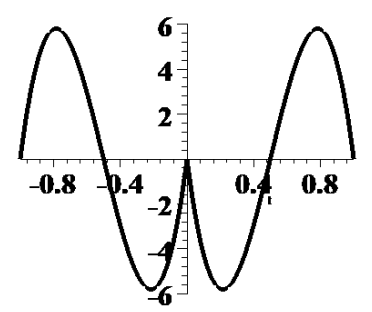

Fig.3. The plot of the $W^{\prime \prime}{ }_{j, 0}$

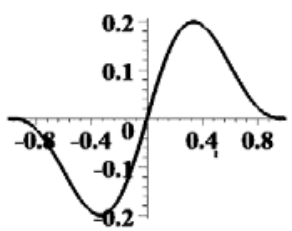

Fig.4. The plot of the $W_{j, 1}$

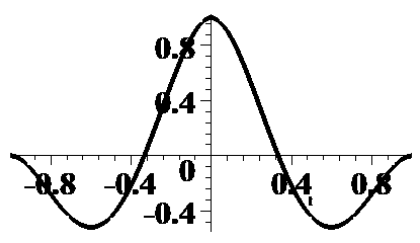

Fig.5. The plot of the $W_{j, 1}^{\prime}$

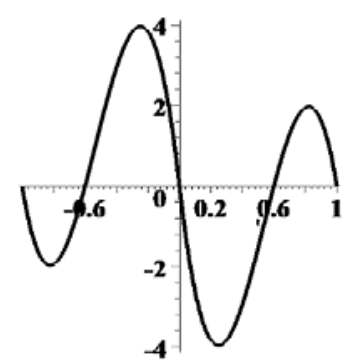

Fig.6. The plot of the $W^{\prime \prime}{ }_{j, 2}$ 


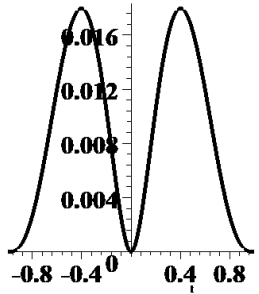

Fig.7. The plot of the $W_{j, 2}$

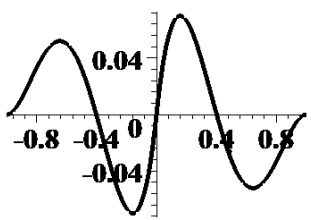

Fig.8. The plot of the $W^{\prime}{ }_{j, 2}$

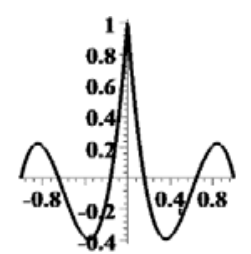

Fig.9. The plot of the $W^{\prime \prime}{ }_{j, 2}$

Let $x \in\left[x_{j}, x_{j+1}\right]$. We construct the approximation in the form:

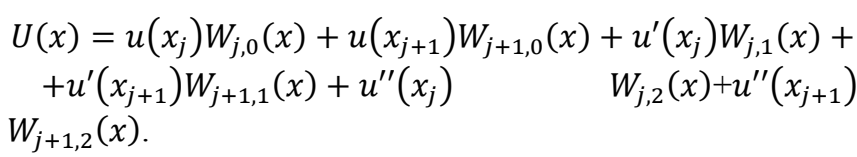

The following theorem is valid if $\varphi_{s}=\cos (s x), s=0,1,2$, $\varphi_{s}=\sin (s x), s=1,2, \varphi_{5}=x$.

Remark. It can be obtained, that when $h \rightarrow 0$ the formulas take the form:

$$
\begin{gathered}
W_{j, 0}\left(x_{j}+t h\right)=-\left(6 t^{2}+3 t+1\right)(t-1)^{3}+O(h), \\
W_{j+1,0}\left(x_{j}+t h\right)=\left(6 t^{2}-15 t+10\right) t^{3}+O(h), \\
W_{j, 1}\left(x_{j}+t h\right)=-t h(3 t+1)(t-1)^{3}+O\left(h^{2}\right), \\
W_{j+1,1}\left(x_{j}+t h\right)=-h(t-1)(3 t-4) t^{3}+O\left(h^{2}\right), \\
W_{j, 2}\left(x_{j}+t h\right)=-h^{2} t^{2}(t-1)^{3} / 2+O\left(h^{3}\right), \\
W_{j+1,2}\left(x_{j}+t h\right)=h^{2} t^{3}(t-1)^{2} / 2+O\left(h^{3}\right),
\end{gathered}
$$

Theorem 2. Let function $u(x)$ be such that $u \in \mathrm{C}^{6}([a, b])$. $\varphi_{i}=x^{i}, i=0,1,2,3,4,5$. Suppose the ordered distinct nodes $x_{k}$ are $x_{k+1}-x_{k}=h, \mathrm{~h}<1.5$. Then for $x \in\left[x_{j}, x_{j+1}\right]$ we have

$$
\begin{aligned}
& |u(x)-U(x)| \leq K_{2} h^{6}\left\|u^{(6)}+5 u^{(4)}+4 u^{(2)}\right\|_{\left[x_{j}, x_{j+1}\right]}, \\
& K_{2}>0
\end{aligned}
$$

Proof. In the non-polynomial case when $x \in\left[x_{j}, x_{j+1}\right]$ we have $W_{i, j}$ which are given above. The method for finding the estimate is described in detail in [11]. Here we briefly dwell on the main points of the proof.

We denote by $\Phi_{i}, i=1,2,3, \ldots 7$, vector columns of the form:

$$
\begin{aligned}
& \Phi_{1}=(1,0,0,0,0,0,0)^{T}, \\
& \Phi_{2}=(\sin (x), \cos (x),-\sin (x),-\cos (x), \sin (x), \cos (x), \\
& -\sin (x))^{T} \\
& \Phi_{3}=(\cos (x),-\sin (x),-\cos (x), \sin (x), \cos (x),-\sin (x), \\
& -\cos (x))^{T} \\
& \Phi_{4}=(\sin (2 x), 2 \cos (2 x),-4 \sin (2 x),-8 \cos (2 x), \\
& 16 \sin (2 x), 32 \cos (2 x),-64 \sin (2 x))^{T}, \\
& \Phi_{5}=(\cos (2 x),-2 \sin (2 x),-4 \cos (2 x), 8 \sin (2 x), \\
& 16 \cos (2 x)-32 \sin (2 x),-64 \cos (2 x))^{T}, \\
& \Phi_{6}=(1, x, 0,0,0,0,0)^{T}, \\
& \Phi_{7}=\left(u, u^{\prime}, u^{\prime \prime}, u^{(3)}, u^{(4)}, u^{(5)}, u^{(6)}\right)^{T} \text {. }
\end{aligned}
$$

Decomposing the determinant

$$
L u=\left(\Phi_{1}, \Phi_{2}, \Phi_{3}, \Phi_{4}, \Phi_{5}, \Phi_{6}, \Phi_{7}\right)
$$

into elements of the last column, we obtain

$L u=5 u^{(4)}+u^{(6)}+4 u^{\prime \prime}$.

Thus we have found a homogeneous equation $L u=$ $5 u^{(4)}+u^{(6)}+4 u^{\prime \prime}=0$.

Now we need to find a solution to the inhomogeneous equation $L u=g(x)$ by the method of varying arbitrary constants.

Let $u(x)=\sum_{1}^{6} C_{i}(x) \varphi_{i}(x)$.

To determine the coefficients $C_{i}(x)$ we should solve a system of linear algebraic equations:

$$
\begin{aligned}
& \sum_{i=1}^{6} C_{i}^{\prime}(x) \varphi_{i}(x)=0, \\
& \sum_{i=1}^{6} C_{i}^{\prime}(x) \varphi_{i}^{(k)}(x)=0, k=1,2,3,4, \\
& \sum_{i=1}^{6} C_{i}^{\prime}(x) \varphi_{i}^{(5)}(x)=g(x) .
\end{aligned}
$$

We denote by $\Psi_{i}, i=1,2,3, \ldots 6$, vector columns of the form: $\Psi_{1}=(1,0,0,0,0,0)^{T}$,

$\Psi_{2}=(\sin (x), \cos (x),-\sin (x),-\cos (x), \sin (x), \cos (x))^{T}$, $\Psi_{3}$

$$
=(\cos (x),-\sin (x),-\cos (x), \sin (x), \cos (x),-\sin (x))^{T},
$$

$\Psi_{4}=(\sin (2 x), 2 \cos (2 x),-4 \sin (2 x),-8 \cos (2 x)$,

$$
\begin{gathered}
\Psi_{5}=\left(\cos (2 x), \begin{array}{c}
16 \sin (2 x), 32 \cos (2 x))^{T}, \\
16 \sin (2 x),-4 \cos (2 x), 8 \sin (2 x),
\end{array}\right. \\
\Psi_{6}=(1, x, 0,0,0,0)^{T} .
\end{gathered}
$$

We denote by $W(x)$ the determinant $W=\left|\Psi_{1}, \Psi_{2}, \Psi_{3}, \Psi_{4}, \Psi_{5}, \Psi_{6}\right|$.

Solving this system of equations, we obtain

$$
C^{\prime}{ }_{i}(x)=\frac{W_{6, i}(x) g(x)}{W(x)}
$$

Here $W_{6, i}(x)$ is the algebraic complement of the elements of the $i$-th column of the 7-th row of the determinant $W(x)$.

Thus, $C_{i}(x)=\int_{x_{j}}^{x} \frac{W_{6, i}(x) g(x)}{W(x)}+c_{i}$, where $c_{i}$ is an arbitrary constant.

Using the results from paper [11] we get 


$$
\begin{aligned}
u(x)= & \int_{x_{j}}^{x}\left(5 u^{(4)}(t)+u^{(6)}(t)+4 u^{\prime \prime}(t)\right)\left(\frac{\sin (2 t-2 x)}{24}-\right. \\
& \left.\frac{\sin (t-x)}{3}+\frac{t-x}{4}\right) d t+c_{1}+c_{2} \sin (x)+ \\
& c_{3} \cos (x)+c_{4} \sin (2 x)+c_{5} \cos (2 x)+c_{6} x,
\end{aligned}
$$

where $c_{i}, i=1,2,3,4,5,6$, are some arbitrary constants.

Using the expression $u(x)$ and derivative of it, we receive the estimation of the error of the approximation with the nonpolynomial splines. The proof is complete.

Note, that when $u(x)=x-\cos (x)-\sin (x)+\frac{x^{2}}{8}+1-$ $-\frac{\sin (2 x)}{4}-\frac{\cos (2 x)}{4}$ we get $u^{(6)}+5 u^{(4)}+4 u^{(2)}=1$.

Remark. It can be calculated that if $h \leq 1$ then $K \approx 0.4$. $10^{-4}$.

The details will be discussed in another paper.

Now we can construct the piecewise function $V(x), x \in$ $[a, b], \quad$ such that $V(x)=U(x)$ for $x \in\left[x_{j}, x_{j+1}\right]$. This piecewise function $V(x)$ interpolates the function $u(x)$ and the first and the second derivative at the nodes. Thus, $V(x)$ is a continuous function and the first and the second derivatives are also continuous ones. So we need the values of the first and the second derivatives of $u(x)$ at the nodes for the construction of the approximation.

Let $h=0.1,[a, b]=[-1,1]$. We denote by $R=\max _{[-1,1]} \mid u-$ $V\left|, R_{1}=\max _{[-1,1]}\right| u^{\prime}-V^{\prime}\left|, R_{2}=\max _{[-1,1]}\right| u^{\prime \prime}-V^{\prime \prime} \mid$. Actual errors in absolute values of the approximation with the non-polynomial splines are given in Table 1. The actual errors in absolute values of the approximation with the polynomial splines are given in Table 2. The theoretical errors in absolute values of the approximation with the polynomial splines are given in Table 3. The theoretical errors in absolute values of the approximation with the non-polynomial splines are given in Table 4.

Table 1. Actual errors in absolute values of the approximation with the non-polynomial splines

\begin{tabular}{|c|c|c|c|}
\hline$u(x)$ & $R$ & $R_{1}$ & $R_{2}$ \\
\hline $\begin{array}{l}1 /(1 \\
\left.+25 x^{2}\right)\end{array}$ & $0.172 \cdot 10^{-4}$ & $0.540 \cdot 10^{-3}$ & $0.399 \cdot 10^{-1}$ \\
\hline $\begin{array}{l}\sin (7 x) \\
-\cos (9 x)\end{array}$ & $0.122 \cdot 10^{-4}$ & $0.353 \cdot 10^{-3}$ & $0.222 \cdot 10^{-1}$ \\
\hline$x^{7}-x^{9}$ & $0.120 \cdot 10^{-5}$ & $0.276 \cdot 10^{-4}$ & $0.191 \cdot 10^{-2}$ \\
\hline
\end{tabular}

Table 2. The actual errors in absolute values of the approximation with the polynomial splines

\begin{tabular}{|c|c|c|c|}
\hline$u(x)$ & $R$ & $R_{1}$ & $R_{2}$ \\
\hline $\begin{array}{c}1 /(1 \\
\left.+25 x^{2}\right)\end{array}$ & $0.169 \cdot 10^{-4}$ & $0.545 \cdot 10^{-3}$ & $0.410 \cdot 10^{-1}$ \\
\hline $\begin{array}{c}\sin (7 x) \\
-\cos (9 x)\end{array}$ & $0.130 \cdot 10^{-4}$ & $0.377 \cdot 10^{-3}$ & $0.235 \cdot 10^{-1}$ \\
\hline$x^{7}-x^{9}$ & $0.102 \cdot 10^{-5}$ & $0.244 \cdot 10^{-4}$ & $0.166 \cdot 10^{-2}$ \\
\hline
\end{tabular}

Table 3. The theoretical errors in absolute values of the approximation with the polynomial splines

\begin{tabular}{|c|l|}
\hline$u(x)$ & $\mathrm{R}$ \\
\hline $1 /\left(1+25 x^{2}\right)$ & $0.244 \cdot 10^{-3}$ \\
\hline $\sin (7 x)-\cos (9 x)$ & $0.141 \cdot 10^{-4}$ \\
\hline$x^{7}-x^{9}$ & $0.120 \cdot 10^{-5}$ \\
\hline
\end{tabular}

Table 4. The theoretical errors in absolute values of the approximation with the non-polynomial splines

\begin{tabular}{|c|c|}
\hline$u(x)$ & $\mathrm{R}$ \\
\hline $1 /\left(1+25 x^{2}\right)$ & $0.447 \cdot 10^{-3}$ \\
\hline $\sin (7 x)-\cos (9 x)$ & $0.241 \cdot 10^{-4}$ \\
\hline$x^{7}-x^{9}$ & $0.266 \cdot 10^{-5}$ \\
\hline
\end{tabular}

\section{NUMERICAL EXAMLES}

Consider the solution of the following boundary value problem:

$$
u^{\prime \prime}=f(x), \quad u(0)=u(1)=0
$$

where $f(x)=\left(\sin ^{3}(x)\left((\sin (x)-\sin (1))^{3}\right)^{\prime \prime}\right.$.

Divide the interval $[0,1]$ into $n$ parts. Thus, we have constructed a grid of nodes $x_{j}, j=0, \ldots n+1$. We are interested in the values of the function in the internal nodes $x_{j}$, $j=1, \ldots n$. The values of the function in the nodes $x_{0}, x_{n+1}$ we know. In order to solve this problem with variationaldifference method we have to calculate the integrals:

$$
\begin{aligned}
{\left[w_{j, i}, w_{j, i}\right] } & =\int_{x_{j-1}}^{x_{j+1}}\left(w_{j, i}^{\prime}\right)^{2} d x, i=0,1,2, \\
{\left[w_{j, i}, w_{j+1, i}\right] } & =\int_{x_{j}}^{x_{j+1}} w_{j, i}^{\prime} w_{j+1, i}^{\prime} d x, i=0,1,2, \\
{\left[w_{j, i}, w_{j+1, k}\right] } & =\int_{x_{j}}^{x_{j+1}} w_{j, i}^{\prime} w_{j+1, k}^{\prime} d x .
\end{aligned}
$$

Next, we need to solve the system of equations $M C=F$.The matrix of the system of equations will have the form:

$$
\left(\begin{array}{lll}
M_{11} & M_{12} & M_{13} \\
M_{21} & M_{22} & M_{23} \\
M_{31} & M_{32} & M_{33}
\end{array}\right) .
$$

The matrices $M_{i j}, i, j=1,2,3$, have a tridiagonal structure. So, for example, the matrix $M_{11}$ has the form:

$$
\left(\begin{array}{ccc}
{\left[w_{j-1,0}, w_{j-1,0}\right]} & {\left[w_{j-1,0}, w_{j, 0}\right]} & 0 \\
{\left[w_{j, 0}, w_{j-1,0}\right]} & {\left[w_{j, 0}, w_{j, 0}\right]} & {\left[w_{j, 0}, w_{j+1,0}\right]} \\
0 & {\left[w_{j+1,0}, w_{j, 0}\right]} & {\left[w_{j+1,0}, w_{j+1,0}\right]}
\end{array}\right)
$$

The right side $F$ of the system $M C=F$ has the form

$$
F=\left(F_{1}, F_{2}, F_{3}\right),
$$

where the elements of $F_{k}, k=1,2,3$, are as followed:

$$
F_{k}=\int_{x_{i-1}}^{x_{i+1}} f(x) w_{i, k} d x
$$


Having solved the system of equations we find the unknown $C=\left(C_{1}, C_{2}, C_{3}\right)$, where $C_{i}=c_{1, i}, \ldots, c_{n, i}$. Next, we construct the solution on each interval separately in the form

$$
\begin{aligned}
& U(x)=c_{j, 0} w_{j, 0}(x)+c_{j+1,0} w_{j+1,0}(x)+c_{j, 1} w_{j, 1}(x)+ \\
& +c_{j+1,1} w_{j+1,1}(x)+c_{j, 2} w_{j, 2}(x)+c_{j+1,2} w(x) .
\end{aligned}
$$

By the construction, the function $U(x)$ is a twice continuously differentiable function. Note that we obtain an approximation to the first and second derivatives of the solution in the form:

$$
\begin{aligned}
& U^{\prime}(x)=c_{j, 0} w_{j, 0}^{\prime}(x)+c_{j+1,0} w_{j+1,0}^{\prime}(x)+c_{j, 1} w_{j, 1}^{\prime}(x)+ \\
& +c_{j+1,1} w^{\prime}{ }_{j+1,1}(x)+c_{j, 2} w_{j, 2}^{\prime}(x)+c_{j+1,2} w_{j+1,2}^{\prime}(x), \\
& U^{\prime \prime}(x)=c_{j, 0} w_{j, 0}^{\prime \prime}(x)+c_{j+1,0} w^{\prime \prime}{ }_{j+1,0}(x)+c_{j, 1} w^{\prime \prime}{ }_{j, 1}(x)+ \\
& +c_{j+1,1} w^{\prime \prime}{ }_{j+1,1}(x)+c_{j, 2} w_{j, 2}^{\prime \prime}(x)+c_{j+1,2}{w^{\prime \prime}}_{j+1,2}(x) .
\end{aligned}
$$

Example 1. Let $u=\sin ^{3}(x)(\sin (x)-\sin (1))^{3}, f(x)=$ $u^{\prime \prime}(x)$. Let $n=10$. Figure 10 shows the plot of the solution of the boundary value problem and the approximate solution when the polynomial splines were used. Figure 11 shows the plot of the first derivative of the solution of the boundary value problem and the first derivative of the approximate solution. Figure 12 shows the plot of the second derivative of the solution of the boundary value problem and the second derivative of the approximate solution.

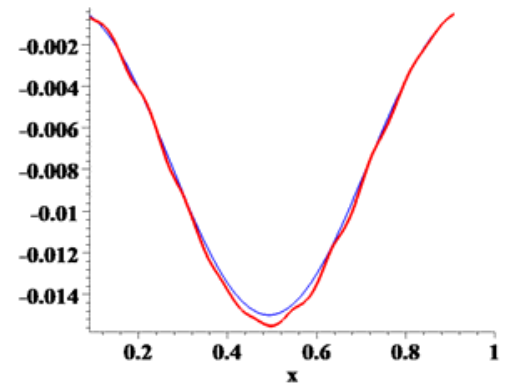

Figure 10. The plot of the solution of the boundary value problem and the approximate solution.

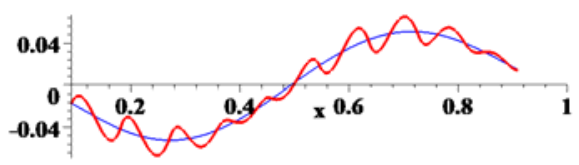

Figure 11. The plot of the first derivative of the solution of the boundary value problem and the first derivative of the approximate solution.

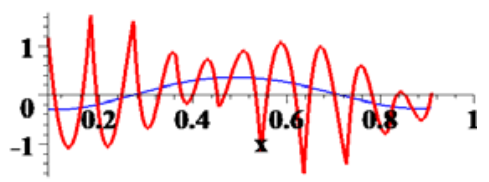

Figure 12. The plot of the second derivative of the solution of the boundary value problem and the second derivative of the approximate solution.

It should be noted that if at the ends of the interval $[a, b]$ the solution of the equation and the first two derivatives of the solution equal to zero, then the difference method can give an approximate solution better than the variationaldifference method. The first example illustrates this situation (see Fig.13).

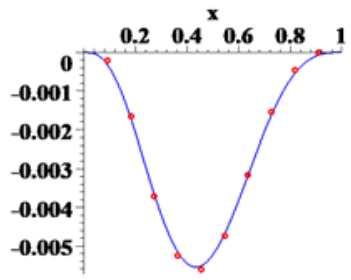

Figure 13. The plots of the solution of this problem using the difference method (points) and the solution $u(x)$ (blue line).

Now consider the case when only the solution equals to zero at the ends of the interval $[a, b]$.

Example 2. Now let $u=x(x-1) \sin \left(\frac{x}{\pi 2}\right), f(x)=(u(x))^{\prime \prime}$, $n=10$, Figure 14 shows the plot of the solution of the boundary value problem and the approximate solution when the polynomial splines were used. Figure 15 shows the plot of the first derivative of the solution of the boundary value problem and the first derivative of the approximate solution. Figure 16 shows the plot of the second derivative of the solution of the boundary value problem and the second derivative of the approximate solution.

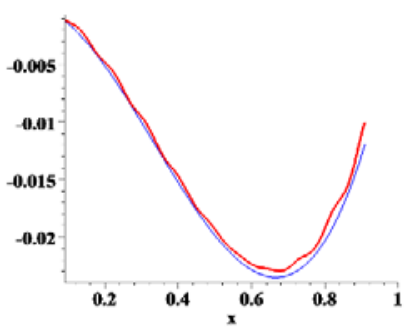

Figure 14. The plot of the solution of the boundary value problem and the approximate solution.

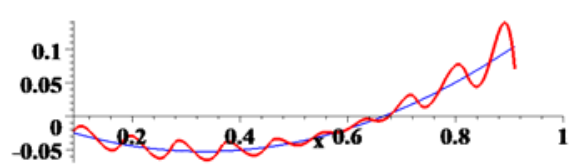

Figure 15. The plot of the first derivative of the solution of the boundary value problem and the first derivative of the approximate solution. 


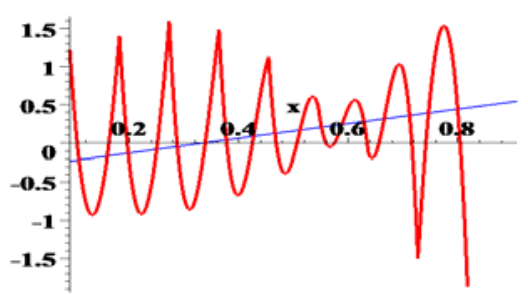

Figure 16. The plot of the second derivative of the solution of the boundary value problem and the second derivative of the approximate solution.

Remark. The result of application of the is better variational-difference method than when we use the difference method with the second order approximation, when $h=0.01$.

The approximate solution of this problem using the difference method with the second order approximation (points) and $u(x)$ are given in Fig. 17 .

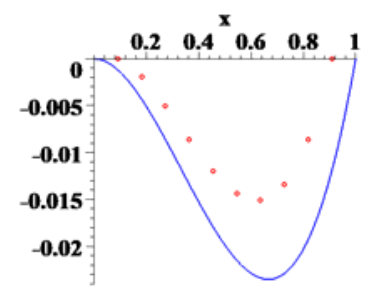

Figure 17. The plots of the solution of this problem using the difference method (points) and the solution $u(x)$ (blue line).

\section{THREE TIMES CONTINUOUSLY DIFFERENTIABLE APPROXIMATION}

Now we consider the question of whether it is possible to obtain an approximation of three times continuously differentiable using the obtained basis functions. We have obtained the function $U(x), x \in\left[x_{j}, x_{j+1}\right]$, such that

$$
\begin{aligned}
& u\left(x_{j}\right)=U\left(x_{j}\right), u^{\prime}\left(x_{j}\right)=U^{\prime}\left(x_{j}\right), u^{\prime \prime}\left(x_{j}\right)=U^{\prime \prime}\left(x_{j}\right), \\
& u\left(x_{j+1}\right)=U\left(x_{j+1}\right), u^{\prime}\left(x_{j+1}\right)=U^{\prime}\left(x_{j+1}\right), \\
& u^{\prime \prime}\left(x_{j+1}\right)=U^{\prime \prime}\left(x_{j+1}\right),
\end{aligned}
$$

using the formula:

$$
\begin{aligned}
& U(x)=u\left(x_{j}\right) w_{j, 0}(x)+u\left(x_{j+1}\right) w_{j+1,0}(x)+u^{\prime}\left(x_{j}\right) w_{j, 1}(x)+ \\
& \quad+u^{\prime}\left(x_{j+1}\right) w_{j+1,1}(x)+u^{\prime \prime}\left(x_{j}\right) w_{j, 2}(x)+u^{\prime \prime}\left(x_{j+1}\right) w_{j+1,2}(x)
\end{aligned}
$$

on every interval $x \in\left[x_{j}, x_{j+1}\right]$.

Consider the algorithm for constructing a thrice continuously differentiable polynomial spline $P(x)$. To construct a thrice continuously differentiable non-polynomial spline, the construction is similar.

Our aim is to construct a piecewise function $P(x)$ so that it will not only have the first and the second continuous derivative but also the third continuous derivative. Moreover, it will interpolate the function $u(x)$ and its first derivative in the nodes $x_{j}$. The way of constructing such piecewise functions in a polynomial case is known (see, for example, papers of Kvasov B.I., Zavyalov Yu.S., Miroshnichenko V.L.).
We shall construct a piecewise function $P(x), \quad x \in$ $[a, b]$,which equals to

$$
\begin{aligned}
& u\left(x_{j}\right) w_{j, 0}(x)+u\left(x_{j+1}\right) w_{j+1,0}(x)+u^{\prime}\left(x_{j}\right) w_{j, 1}(x)+ \\
+ & u^{\prime}\left(x_{j+1}\right) w_{j+1,1}(x)+c_{j} w_{j, 2}(x)+c_{j+1} w_{j+1,2}(x) .
\end{aligned}
$$

on every $x \in\left[x_{j}, x_{j+1}\right]$. The parameters $c_{j}, c_{j+1}$ are defined by the condition that the third derivative of $P(x)$ is continuous. The piecewise approximation $P(x)$ will be such that it is continuous and the first two derivatives of the piecewise interpolation will also be continuous.

Let $c_{j-1}, c_{j}, c_{j+1}$ be some parameters to be determined, and $x=x_{j}+t h, \quad t \in[0,1]$. On every interval $\left[x_{j}, x_{j+1}\right], j=$ $0, \ldots, n-1$, we construct the approximation in the form:

$$
\begin{gathered}
P_{j}(x)=c_{j} w_{j, 2}(t)+c_{j+1} w_{j+1,2}(t)+u\left(x_{j}\right) w_{j, 0}(t)+ \\
u\left(x_{j+1}\right) w_{j+1}(t)+u^{\prime}\left(x_{j}\right) w_{j, 1}(t)+u^{\prime}\left(x_{j+1}\right) w_{j+1,1}(t) .
\end{gathered}
$$

We differentiate three times this expression and the similar one when $x \in\left[x_{j-1}, x_{j}\right]$. After that, set them equal to each other in the common node $x_{j}, j=1, \ldots, n-1$. Thus we construct the equation:

$$
\begin{gathered}
c_{j-1} w^{\prime \prime \prime}{ }_{j-1,2}(1)+c_{j} w^{\prime \prime \prime}{ }_{j, 2}(1)+ \\
u\left(x_{j-1}\right) w^{\prime \prime \prime}{ }_{j-1,0}(1)+u\left(x_{j+1}\right) w^{\prime \prime \prime}{ }_{j, 0}(1)+ \\
u^{\prime}\left(x_{j-1}\right) w^{\prime \prime \prime}{ }_{j-1,1}(1)+u^{\prime}\left(x_{j+1}\right) w^{\prime \prime \prime}{ }_{j, 1}(1)= \\
c_{j+1} w^{\prime \prime \prime}{ }_{j+1,1}(0)+c_{j} w^{\prime \prime \prime}{ }_{j, 1}(0)+ \\
u\left(x_{j}\right) w^{\prime \prime \prime}{ }_{j, 0}(0)+u\left(x_{j+1}\right) w^{\prime \prime \prime}{ }_{j+1,0}(0)++ \\
u^{\prime}\left(x_{j}\right) w^{\prime \prime \prime}{ }_{j, 1}(0)+u^{\prime}\left(x_{j+1}\right) w^{\prime \prime \prime}{ }_{j+1,1}(0),+
\end{gathered}
$$

where functions $w_{j-1,2}\left(x_{j-1}+t h\right), w_{j, 2}\left(x_{j-1}+t h\right)$,

$w_{j-1,1}\left(x_{j-1}+t h\right), w_{j, 1}\left(x_{j-1}+t h\right), w_{j-1,0}\left(x_{j-1}+t h\right)$, $w_{j, 0}\left(x_{j-1}+t h\right), \quad$ constructed when $x_{j-1}+t h=x, \quad x \in$ $\left[x_{j-1}, x_{j}\right]$. We need two extra conditions at the ends of the interval $[a, b]$. Let $P_{j}^{\prime \prime}(a)=u^{\prime \prime}(a)$ and $P_{j}^{\prime \prime}(b)=u^{\prime \prime}(b)$.

Taking into account the interpolation conditions $P_{j}\left(x_{j}\right)=$ $u\left(x_{j}\right), \quad P_{j}^{\prime}\left(x_{j}\right)=u^{\prime}\left(x_{j}\right), \quad j=0, \ldots, n$, and the boundary conditions $P_{j}^{\prime \prime}\left(x_{j}\right)=u^{\prime \prime}\left(x_{j}\right), j=0, n$, we construct the piecewise function $Q(x)$. This function and its first two derivatives will be continuous. It interpolates the function $u$ in the nodes $x_{j}, j=0, \ldots, n$. So we need the expressions for the third derivative of the basis functions $w_{j, i}$. The third derivative of the basis functions $w_{j, i}$. can be easily obtained. Thus we have to solve the system of algebraic equations $G C=$ $F$, where the square matrix $G=\{g\}_{i, j}^{n-1}, F=\{f\}_{j=1}^{n-1}$, where

$$
\begin{gathered}
f_{1}=-\left(u\left(x_{1}\right) Y_{j}+u\left(x_{2}\right) Y_{j+1}+u\left(x_{0}\right) Y_{j-1}+\right. \\
\left.u^{\prime}\left(x_{1}\right) Y_{j}^{1}+u^{\prime}\left(x_{2}\right) Y_{j+1}^{1}+u^{\prime}\left(x_{0}\right) Y_{j-1}^{1}\right)-u^{\prime \prime}\left(x_{0}\right) C_{j+1}, \\
f_{n-1}=-\left(u\left(x_{n-1}\right) Y_{j}+u\left(x_{n}\right) Y_{j+1}+u\left(x_{n-2}\right) Y_{j-1}+\right.
\end{gathered}
$$




$$
\begin{gathered}
\left.u^{\prime}\left(x_{n-1}\right) Y_{j}^{1}+u^{\prime}\left(x_{n}\right) Y_{j+1}^{1}+u^{\prime}\left(x_{n-2}\right) Y_{j-1}^{1}\right)-u^{\prime \prime}\left(x_{n}\right) C_{j+1}, \\
f_{j}=-\left(u\left(x_{j}\right) Y_{j}+u\left(x_{j+1}\right) Y_{j+1}+u\left(x_{j-1}\right) Y_{j-1}+\right. \\
u^{\prime}\left(x_{j}\right) Y_{j}^{1}+u^{\prime}\left(x_{j+1}\right) Y_{j+1}^{1}+u^{\prime}\left(x_{j-1}\right) Y_{j-1}^{1}, \\
j=2, \ldots, n-2 .
\end{gathered}
$$

We have $g_{j, j}=c_{j}, \quad g_{j, j+1}=c_{j+1}, g_{j-1, j}=c_{j-1}$. We obtain the following expressions for the polynomial case with equidistant nodes: $c_{j}=9 / 2, c_{j+1}=-3 / 4, c_{j-1}=-3 / 4, Y_{j}=$ $\frac{30}{h^{2}}, Y_{j+1}=-\frac{15}{h^{2}}, Y_{j-1}=-15 / h^{2}, Y_{j}^{1}=0, Y_{j+1}^{1}=6 / h, Y_{j-1}^{1}=$ $-6 / h$.

Using the expression on every interval $\left[x_{j}, x_{j+1}\right]$

$$
\begin{gathered}
P_{j}(x)=c_{j} w_{j, 2}(t)+c_{j+1} w_{j+1,2}(t)+u\left(x_{j}\right) w_{j, 0}(t)+ \\
u\left(x_{j+1}\right) w_{j+1}(t)+u^{\prime}\left(x_{j}\right) w_{j, 1}(t)+u^{\prime}\left(x_{j+1}\right) w_{j+1,1}(t)
\end{gathered}
$$

we obtain the smooth approximation.

Let $h=0.1,[a, b]=[-1,1]$. Figure 18 shows the plot of the error of the approximation of the third derivative of the function $u(x)=\sin (3 x)+\cos (2 x)$ with the polynomial splines $U(x)$. This plot is discontinuous. Figure 19 shows the plot of the error of the approximation of the third derivative of the function $u(x)=\sin (3 x)+\cos (2 x)$ with the polynomial splines $Q(x)$. Figure 20 shows the plot of the error of the approximation of the second derivative of the function $u(x)=$ $\sin (3 x)+\cos (2 x)$ with the polynomial splines $Q(x)$. Figure 21 shows the plots of the error of the approximation of the function $u(x)=\sin (3 x)+\cos (2 x)$ with the polynomial splines $Q(x)$ and $U(x)$. Figure 22 shows the plots of the error of the approximation of the first derivative of the function $u(x)=\sin (3 x)+\cos (2 x)$ with the polynomial splines $Q(x)$ (thick blue) and $U(x)$ (thin red).

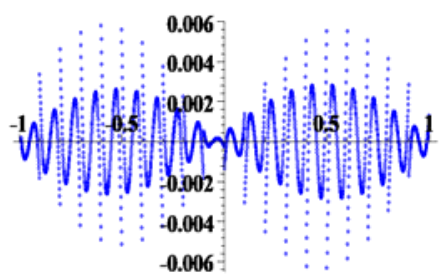

Figure 18. The plot of the error of the approximation with splines $U(x)$ of the third derivative of the function $u(x)=\sin (3 x)+\cos (2 x)$.

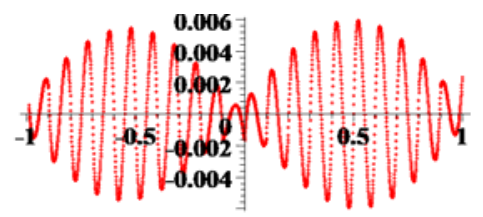

Figure 19. The plot of the error of the approximation with splines $Q(x)$ of the third derivative of the function $u(x)=$ $\sin (3 x)+\cos (2 x)$.

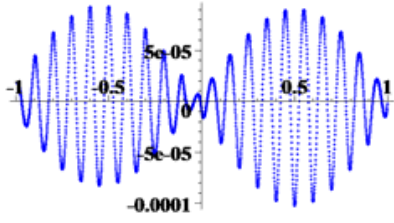

Figure 20. The plot of the error of the approximation of the second derivative of the function $u(x)=\sin (3 x)+\cos (2 x)$.

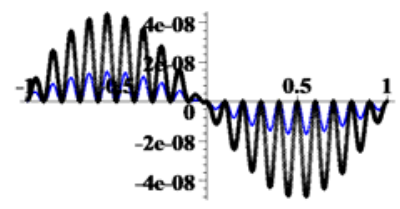

Figure 21. The plots of the error of the approximation of the function $u(x)=\sin (3 x)+\cos (2 x)$ with the polynomial splines $Q(x)$ (thick black) and $U(x)$ (thin blue).

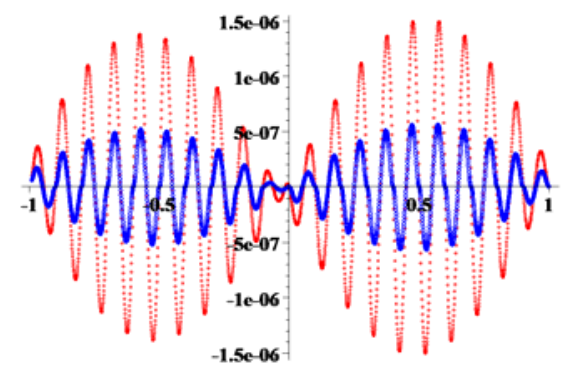

Figure 22. The plots of the error of the approximation of the first derivative of the function $u(x)=\sin (3 x)+\cos (2 x)$ with the polynomial splines $Q(x)$ (thick blue) and $U(x)$ (thin red).

We obtain the following expressions for the non-polynomial case with equidistant nodes:

$$
\begin{gathered}
Y_{j-1}=-h\left(4 \cos (h)-2 \cos ^{2}(h)-2\right) /\left(4 \left(h \cos ^{2}(h)\right.\right. \\
-3 \sin (h) \cos (h)+h \cos (h)-2 h \\
+3 \sin (h))), \\
Y_{j}=-h\left(-8 \cos (h)+4 \cos ^{2}(h)+4\right) /\left(4 \left(h \cos ^{2}(h)\right.\right. \\
-3 \sin (h) \cos (h)+h \cos (h)-2 h \\
+3 \sin (h)), \\
\quad \\
Y_{j+1}=-h\left(4 \cos (h)-2 \cos ^{2}(h)-2\right) /\left(4 \left(h \cos ^{2}(h)\right.\right. \\
-3 \sin (h) \cos (h)+h \cos (h)-2 h \\
+3 \sin (h))), \\
Y_{j+1}^{1}=-h(6 \sin (h)-3 h-6 h \cos (h)+3 \sin (h) \cos (h)) / \\
\left(4 \left(h \cos ^{2}(h)-3 \sin (h) \cos (h)+h \cos (h)-2 h\right.\right. \\
+3 \sin (h))), \\
Y_{j-1}^{1}=-h(-6 \sin (h)+3 h+6 h \cos (h) \\
-3 \sin (h) \cos (h)) / \\
\left(4\left(h \cos ^{2}(h)-3 \sin (h) \cos (h)+h \cos (h)-2 h+3 \sin (h)\right)\right),
\end{gathered}
$$




$$
c_{j}=-h\left(-8 \cos (h)+16 \cos ^{2}(h)+6 h \sin (h) \cos (h)+\right.
$$$$
6 h \sin (h)-8) /\left(4 \left(h \cos ^{2}(h)-3 \sin (h) \cos (h)+h \cos (h)-\right.\right.
$$
$2 h+3 \sin (h)))$,

$$
\begin{gathered}
c_{j+1}=-h\left(4 \cos (h)+\cos ^{2}(h)+3 h \sin (h)\right. \\
-5) /\left(4 \left(h \cos ^{2}(h)-3 \sin (h) \cos (h)\right.\right. \\
+h \cos (h)-2 h+3 \sin (h))), \\
c_{j-1}=-h\left(4 \cos (h)+\cos ^{2}(h)+3 h \sin (h)\right. \\
-5) /\left(4 \left(h \cos ^{2}(h)-3 \sin (h) \cos (h)\right.\right. \\
+h \cos (h)-2 h+3 \sin (h))) .
\end{gathered}
$$

Table 5 shows the actual errors of the smooth polynomial and non-polynomial approximations, $h=0.4,[a, b]=[-1,1]$.

Table 5. The actual errors of the smooth polynomial and non-polynomial approximations, $h=0.4,[a, b]=[-1,1]$.

\begin{tabular}{|c|c|c|}
\hline Function $u(x)$ & $\begin{array}{l}\text { Polynomial } \\
\text { splines }\end{array}$ & $\begin{array}{l}\text { Non-polynomial } \\
\text { splines }\end{array}$ \\
\hline $\sin (3 x)$ & $0.772 \cdot 10^{-4}$ & $0.387 \cdot 10^{-4}$ \\
\hline $\begin{array}{c}\sin (7 x) \\
-\cos (9 x)\end{array}$ & $0.249 \cdot 10^{-1}$ & $0.236 \cdot 10^{-1}$ \\
\hline$x^{7}-x^{9}$ & $0.110 \cdot 10^{-2}$ & $0.121 \cdot 10^{-2}$ \\
\hline $1 /\left(1+25 x^{2}\right)$ & $0.358 \cdot 10^{-1}$ & $0.361 \cdot 10^{-1}$ \\
\hline
\end{tabular}

\section{REFERENCES}

[1] S.G.Mikhlin. "Variational-difference approximation," J. Math. Sci, Vol.10, 1978, pp. 661-787.

[2] E.L.Hart, V.S. Hudramovich, "Application of the Projection-Iterative Scheme of the Method of Local Variations to Solving Stability Problems for Thin-Walled Shell Structures Under Localized Actions," Strength of Materials, 50 (6), 2018, pp. 852-858.

[3] R.Pasichnyk, O.Pasichnyk, O.Uzhegova, O.Andriichuk, O. Bondarskii, "Calculation optimization of complex shape shells by numerical method," Lecture Notes in Mechanical Engineering, 2020 pp. 643-652.

[4] M.N.Suardi, N.Z.F.M.Radzuan, J.Sulaiman, "Performance of quartersweep SOR iteration with cubic B-spline scheme for solving two-point boundary value problems," Journal of Engineering and Applied Sciences, 14 (3), 2019, pp. 693-700.

[5] M.Kang, J.Yang, X.Wang, X.Chen, "Study on the variationaldifference-based design and slow tool servo turning of progressive addition lenses", Advances in Mechanical Engineering, 2018,10(12).

[6] M.S.Korytov, V.S.Shcherbakov, V.V.Titenko,"Application of Hermite splines for load movement on a flexible crane suspension through a curvilinear trajectory," Journal of Physics: Conference Series, 1441 (1), paper № 012101, 2020.

[7] Y.K.Dem'Yanovich, O.V. Belyakova, B.T.N.Le, "Uniqueness of Space of Hermite Type Splines," Proceedings 2018 International Conference on Applied Mathematics and Computational Science, ICAMCS.NET 2018, paper № 8955759, 2018, pp. 178-183.

[8] E.B. Chin, N.Sukumar, "Modeling curved interfaces without elementpartitioning in the extended finite element method," International Journal for Numerical Methods in Engineering, vol. 120, no.5, 2019, pp. 607-649.

[9] N.C. Gabrielides, N.S. Sapidis, "Cubic polynomial and cubic rational C 1 sign,monotonicity and convexity preserving Hermite interpolation," Journal of Computational and Applied Mathematics, vol. 357, 2019, pp. 184-203.

[10] I.J.Schoenberg, "On trigonometric spline interpolation," J Math Mech., 13,1964 , pp. $795--825$.
[11] Irina Burova, "On left integro-differential splines and Cauchy problem," International Journal of Mathematical Models and Methods in Applied Sciences, Vol. 9, 2015, pp. 683-690.

[12] I.G.Burova, "On trigonometric splines construction," Vestnik SanktPeterburgskogo Universiteta, Ser 1. Matematika Mekhanika Astronomiya, 2, 2004, pp. 9-14.

[13] I.G.Burova, T.O.Evdokimova, "On the smooth second order trigonometric splines,"'Vestnik Sankt-Peterburgskogo Universiteta. Ser 1. Matematika Mekhanika Astronomiya, 3, 2004, pp. 11--16.

[14] I.G. Burova, E.F. Muzafarova, I.I. Narbutovskikh, ”Approximation by the Third-Order Splines on Uniform and Non-uniform Grids and Image Processing," WSEAS Transactions on Mathematics, Vol. 19, 2020, pp. $65-73$.

[15] I. G. Burova, E. F. Muzafarova, "Interval Estimation using IntegroDifferential Splines of the Third Order of Approximation," WSEAS Transactions on Mathematics, Vol. 18, 2019, pp. 153-160. 